\title{
Forest Fire Detection System using LoRa Technology
}

\author{
Nicoleta Cristina GAITAN ${ }^{1}$, Paula HOJBOTA ${ }^{2}$ \\ Faculty of Electrical Engineering and Computer Science, Stefan cel Mare University of Suceava ${ }^{1,2}$ \\ Integrated Center for Research, Development and Innovation in Advanced Materials, Nanotechnologies \\ and Distributed Systems for Fabrication and Control (MANSiD) Suceava, Romania ${ }^{1}$
}

\begin{abstract}
Millions of hectares of forest worldwide are affected annually by fires, which can lead to the loss of human lives, materials, destruction of natural flora and fauna but also can lead to the losses of raw materials. The problem is even greater in forests that are not guarded and do not have communication systems available. Thus, in recent years, have been proposed various systems that use devices based on Internet of Things (IoT) for real-time forest fire detection. In this paper, it is proposed a system capable of quickly detecting forest fires on long wide distance. In the development of this system it is used LoRa (Long Range) technology based on LoRaWAN (Long Range Wide Area Network) protocol which is capable to connect low power devices distributed on large geographical areas being an innovative and great solution for transmissions of a low data transfer rate and a low transmission power on high ranges, and because has a great efficiency.
\end{abstract}

Keywords-LoRa; real-time; long range wide area network; internet of things

\section{INTRODUCTION}

Forest fires that take place in the warm season can be caused or started by natural events or human negligence. Natural events such as burning branches or dry leave appear due to the heat generated by the sun. An example of such event is the fire from Siberia in the summer of the year 2019, resulting in the destruction of millions of hectares of vegetation. The fires generated by human negligence can result from multiple factors, such as leaving unattended fires in the forest, throwing a burning cigar etc. An example of a forest fire started due to human negligence took place in the province of South Sumatra, in 2015.

In the modern era, the technology has developed so much that it's become more and more sophisticated, making wireless transmissions real easy. The network of wireless sensors, named WSN (Wireless Sensors Network), based on the LoRa module, can be a great alternative for detecting forest fires in specific areas.

LoRa (Long Range) is a low power technology developed by Semtech and supported by LoRa Alliance. This technology is characterized by a low data transfer rate and a low transmission power on high ranges [1][2]. While the transmission range grows, LoRa keeps its low power characteristics of modulation in frequency (Frequency Shift Keying). This technology modulates symbols with a bandwidth of 125,250 and $500 \mathrm{kHz}$ (in case of European applications) with different spreading factors [3].
The LoRa Alliance defines two different layers of this technology: LoRa physical layer and LoRaWAN (Long Range Wide Area Network) protocol.

From the physical layer point of view, LoRa is a radio modulation patented technique by Semtech. In this case, the technology is going to work with frequencies under the order of $\mathrm{GHz}$ in the unlicensed ISM (Industrial, Scientific and Medical) band [2].

LoRaWAN protocol is standardized by the LoRa Alliance, being defined as a MAC layer protocol and a system architecture that uses LoRa's physical layer. The access control mechanism to environment offered by LoRa's allows for multiple final devices to communicate with a gateway using LoRa modulation (see Fig. 1) [2].

The Low-Power Wide-Area network ensures the connectivity of low power devices distributed on large geographical areas. These networks represent a new model of communication, successfully completing the already existent wireless communication technology such as: Bluetooth, ZigBee, LTE, GSM and Wi-Fi [2].

Thanks to its low power requirements and low costs of manufacturing and operating, LoRaWAN is one of the most used LPWA (Low-Power Wide-Area) technologies.

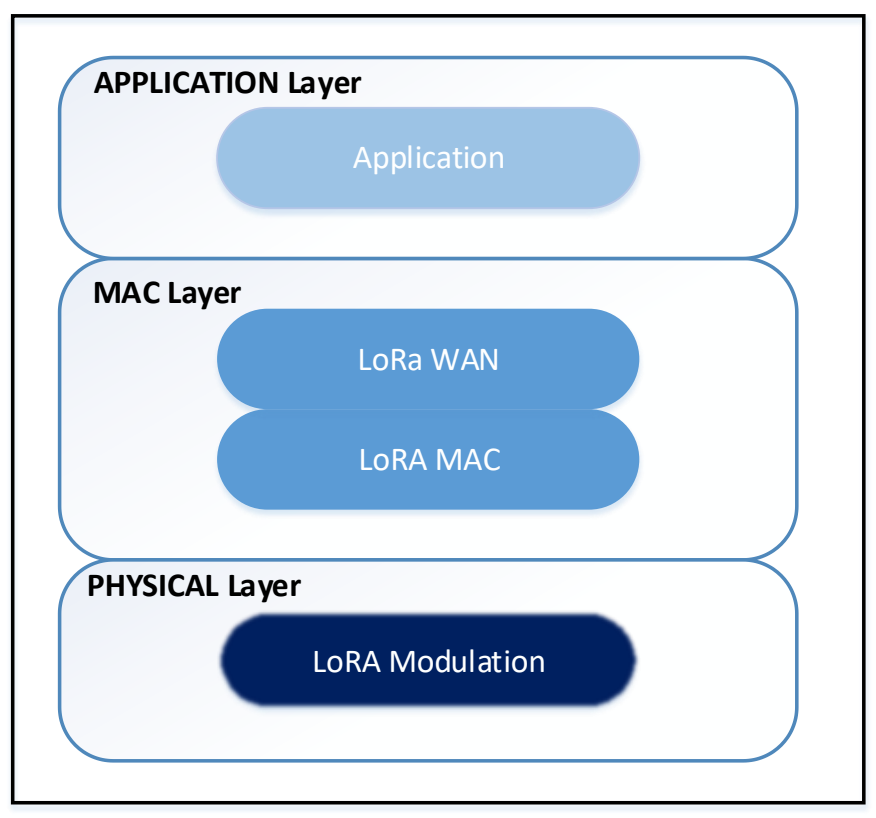

Fig. 1. Long Range Wide Area Network Stack. 
The LoRa technology doesn't guarantee a long time development, but there are already available solutions based on it, unlike other technologies that could possibly disturb the global development [4].

Industrial IoT applications are based on long distance communication. The promising protocols in this domain are SigFox, LoRaWAN and the NB-IoT standard [1]. In Table I is presented a comparison between SigFox and LoRa.

TABLE I. SIGFOX VS. LORA COMPARISON

\begin{tabular}{|l|l|l|}
\hline Parameter & SigFox & LoRa \\
\hline Frequency Band & $868,902[\mathrm{MHz}]$ & $433(\mathrm{US}), 863-870(\mathrm{EU})[\mathrm{MHz}]$ \\
\hline Data Rate & $100 \mathrm{bps}$ & $10 \mathrm{kbps}$ \\
\hline Rural Range & $30-50 \mathrm{~km}$ & $15-20 \mathrm{~km}$ \\
\hline Urban Range & $3-10 \mathrm{~km}$ & $2-5 \mathrm{~km}$ \\
\hline
\end{tabular}

The Internet of Things is a communication model that represents a near future solution, capable of integrating sensors and devices that can communicate directly between them without human intervention. The "things" from Internet of Things include physical devices equipped with microcontrollers, transceivers for digital communications and stacks of protocols for making possible the communication between users [5][6].

With such a great multitude of IoT applications, the LoRaWAN protocol and LoRa devices seems to be very efficient in business, contributing to make people lives well around the globe [4]. Using this technology it expecting to achieve a smart connection of the entire planet. LoRa applications are found in various field, such as, agriculture, smart cities, smart environment, healthcare, smart homes and buildings, industrial control, smart metering, smart supply chain and logistics.

In this article it is presented a study of a system that follows the integration of LoRa technology in forest fire detection.

The rest of the paper it is structured as follows. Section II summarizes some interesting systems focused on fire detectors. The proposed design is presented in Section III. Section IV presents conclusion and future work.

\section{RELATED WORKS}

The traditional methods of forest fire detection and prevention are based on observation through satellite images, visual observation by guards, observation by air or video detection on high ranges [7].

Considering all the disadvantages of conventional forest fire detection systems, in the last years different solutions were proposed in order to improve the monitoring systems of the environment and to create a new real time forest fire detection systems using devices based on the Internet of Things [8].

The detection through IoT based devices and online monitoring systems [9]. In this article, the authors proposed a new forest fire monitoring system by detecting temperature variations and analyzing $\mathrm{CO} 2$ levels. The forest fire detection system was realized using the Arduino Uno module alongside a temperature sensor, a smoke sensor and an alarm system. The temperature sensor detects the variations in temperature and the smoke sensors detect the $\mathrm{CO} 2$ levels and if it is necessary, it enables the alarm (the buzzer on the Arduino Uno board). Using the IoT technology, the system was connected to a webpage named „FireSecurity System”, created in PHP and controlled by the Arduino programming environment [9].

In the article [10], the authors proposed an implementation of a forest fire detection system in the forest area using modern equipment. The system was not only meant for fire detection, but for alarming the forest officer regarding the started fire in the forest. The system's activities are controlled by a microcontroller, and the used sensors have the role of identifying the fire and its exact location. This system is based on IoT, the activities being continuously monitored, and the data being saved and shared to online websites. The forest officer regularly reviews the newly stored data, which can be verified at any time [10]. The monitoring system is usually based near the forest office or a fireman station.

A Smart Forest-Fire Early Detection Sensory System: Another Approach of Utilizing Wireless Sensor and Neural Networks [11]. This article has as its main purpose the implementation of a fast forest fire detection system using cheap and small sizes sensors that do not require surveillance. The system does not utilize large scale centralization systems, which could affect the system's robustness in vulnerable environments. The used sensors network represents a small scale cell that can be multiplied in order to cover the entire forest [11].

Wireless sensor network for forest fire detection [12]. In order to overcome to loss of thousands of forest hectares, the authors propose a forest fire detection system by using a sensor network. Each node has a microcontroller, a transmitter, a receiver and 3 sensors. The measurement methods consist of temperature measurement, detection of methane levels, hydrocarbons and $\mathrm{CO} 2$ levels.

Emerging methods for early detection of forest fires using unmanned aerial vehicles and LoRaWAN sensor networks [13]. In this article, the authors propose 2 different solutions for detecting forest fires. First solution consists of aerial vehicles without pilots (UAVs) equipped with special cameras. In this papers there are presented and analyzed multiple scenarios of drone usages for forest fire detection, including a solution which uses a combination between UAV with fixed-wind and UAV with rotary-wings. The system's basic configuration presented in this article implies using a network of soil mounted cameras through which the constant monitoring of the forest is ensured. The cameras used have double lenses which offer both standard images and infrared images (IR) [13].

In other research paper [14], the authors propose a model of communication based on WSN for detecting forest fires in real time as a much more efficient method of monitoring them than the satellite [15]. Their main purpose is fast detecting of fires in order to reduce the loss of vegetation, flora and fauna. In the realization of this system, it's proposed the usage of some sensors that collect the measured dates and send them to the group of nodes for further processing by building a neuronal network. The neuronal network produces a meteorological 
index that measures the probability of the weather favoring an eventual fire. The cluster headers send meteorological indexes to a node manager that has the role of deciding the potential danger for a fire considering the received indexes [15].

\section{SYSTEM DESCRIPTION}

In this section of the article it is presented the proposed system and different hardware parts which are going to be implemented.

\section{A. General Description}

The capacity to detect if a fire is present is the most important part of a fire safety strategy. Without fire detection means, it cannot alert the population, it cannot take safety measure against the fire and it cannot alarm the firefighting service. The people, by our nature, are capable of feeling heat and smoke, to see the flames and to hear the fire burning. Therefore, we could be great potential fire detectors, but we are not always available or trustworthy, and for this reason, we need to use the technology to replace those abilities.

After analyzing the problems, the losses resulted from forest fires and the high costs of detection and monitoring systems, I believe it is necessary to develop some low cost systems capable of evaluating the risk of a forest fire starting, but also its presence.

The system proposed in this article is realized based on a LoPy4 development board connected to a Pycom Expansion Board 3.0 (Fig. 2), an Arduino Mega 2560 module, a temperature and humidity sensor and a flame detector.

\section{B. Pycom LoPy and Pycom Expansion Board 3.0}

The main component of the proposed forest fire detection system is the LoPy4 development board from Pycom. The LoPy4 is a development platform compatible with MicroPython (WiFi, LoRa, SigFox, and Bluetooth). This board is programmable using MicroPython and the Pymakr plugins for a fast IoT applications development. LoPy4 can be configured in LoRa mode to directly send packages between LoPy4 development boards of the same kind. It represents the best combination of implementing speed and access to the new LPWAN networks from Europe, USA, Africa and India. LoPy4 is CE, FCC, LoRaWAN and SigFox certified [16]. In order for the LoPy4 module to be programmed, we are going to use it along with the PyCom Expansion Board 3.0 development board. This board allows the creation and connection of new IoT projects with WiPy 2.0, WiPy 3.0, LoPy, LoPy4, SiPy, FiPy and Gpy. Pycom Expansion Board 3.0 has different libraries and templates, therefore, the development of an IoT solution becomes much easier and faster [17][20][21][22][23].

\section{Arduino Mega 2560}

Arduino Mega 2560 is a board equipped with a microcontroller and it is based on ATmega2560. The module has 54 pins for digital inputs and outputs (15 of them capable of being used for PWM outputs), 16 analogic inputs, 4 UARTs, a crystal oscillator with a frequency of $16 \mathrm{MHz}$, an USB connection, an ICSP header and a reset button. The Arduino Mega 2560 board can be programmed with the Arduino Software (IDE) [18]. I chose to use the Arduino board because it is easier to use by students and is even cheaper.

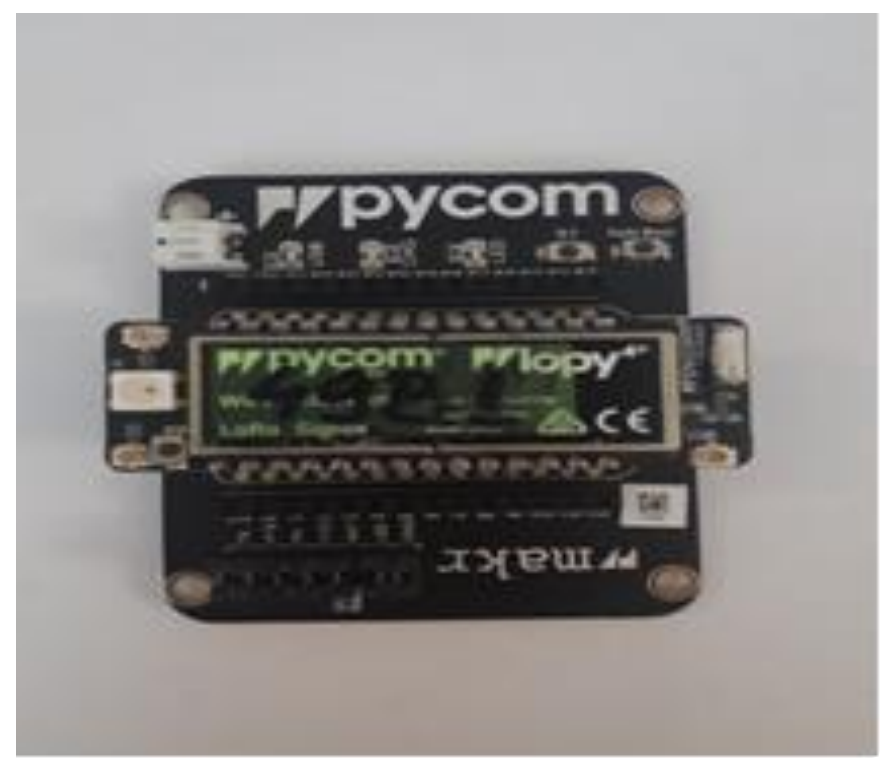

Fig. 2. The Pycom Lopy and Pycom Expansion Board 3.0.

\section{Temperature Sensor DHT11}

The DHT11 is a cheap base sensor, used for measuring temperature and relative humidity at the same time. The measurement of air humidity is ensured by a capacitive humidity sensor, while the temperature is measured using a thermistor, resulting in a digital signal on the data pin. This sensor is a small sized, low power usage, recommended in applications where data acquisition with a high frequency is not required [3]. The sensor operates in cycles of 1 second. In this cycle, the Arduino module and the sensor exchange information about the temperature and humidity. The features of the DHT11 are presented in Table II.

\section{E. Flame Detector}

A flame sensor is a device used to detect the presence of either a fire source or any lightning source. The flame detection can be done from a distance of 1 meter, and the angle detection is 60 degrees. The flame detectors with infrared or with large band monitor the spectral band in infrared for specific models given by hot gasses. These are recognized using a special camera, using thermal imagistic for countering the fires, which is a thermographic camera [19].

TABLE II. DHT11 FEATURES

\begin{tabular}{|l|l|}
\hline DHT11 Specifications & Values \\
\hline Operating Voltage & $3.5 \mathrm{~V}$ to $5.5 \mathrm{~V}$ \\
\hline Operating current & $0.3 \mathrm{~mA}$ (measuring) $60 \mathrm{uA}$ (standby) \\
\hline Output & Serial data \\
\hline Temperature Range & $0^{\circ} \mathrm{C}$ to $50^{\circ} \mathrm{C}$ \\
\hline Humidity Range & $20 \%$ to $90 \%$ \\
\hline Resolution & Temperature and Humidity both are 16 -bit \\
\hline Accuracy & $\pm 1^{\circ} \mathrm{C}$ and $\pm 1 \%$ \\
\hline
\end{tabular}




\section{CONCLUSIONS}

As the IoT technology is being developed more and more, the devices capable of communicating remotely from distance without using a lot of energy have become a necessity more than ever. This challenge of the future is currently served by Long-Power Wide-Area Networks. Currently, there are a lot of applications based on the LPWA technologies, but most of them do not have LoRa module incorporated.

The global warming will constantly be contributing to increasing the number of fires and the damages caused by them. Each season, besides thousands of hectares of forest being destroyed, there are a lot of assets and properties being affected as well. Even more, both the firefighters and the civilians' lives are also in danger. This is the main reason I want to address this issue in this article, for the sole purpose of reducing and preventing devastating fires. The desire to create such a system comes from the fact my father is a forest guard and has been so for more than twenty years. One of his most important responsibilities is to watch out for the forest's well growth and make sure nothing is going to harm it.

In this article, in the Related Works section there were presented different systems of forest fires detection based on LPWA communications. The system proposed in the article is based on the LoRa technology and it has as its main component the LoPy development board from Pycom. The system can be used in extreme temperature conditions, but it can be affected if it's situated in the middle of the fire. With the help of the flame detector, the flames can be noticed from a distance of 100 centimeters. It detects and sends information regarding a possible fire starting out.

Although we know that the most environmental monitoring systems are made using Arduino boards and IoT extensions interfaces, it is desire to emphasize that the novelty of this system is the fact that it is tested with the Arduino board. It is easier to test with the Arduino board because there is online software and it is not expensive.

The decision of using LoRa technology in the proposed system is due of it capabilities to connect low power devices distributed on large geographical areas and because represents an innovative solution for long wide distance transmissions with low power on high ranges consumption and also because has a great efficiency on long-distance data transmission and low transfer power.

For future research, the proposed system can be improved, for example by integrating a surveillance camera for a more precise monitoring process and detection.

Another improvement in the development of this proposed system would be the integration of a GPS module.

\section{REFERENCES}

[1] T. Polonelli, D. Brunella, A. Girolami, G. N. Demmi și L. Benini, „A multi-protocol system forconfigurable data streaming on IoT healthcare devices," 8th International Workshop on Advances in Sensors and Interfaces (IWASI), 2019, pp. 112-117.

[2] L. Leonardi, F. Battaglia, G. Patti și L. L. Bello, „Industrial LoRa: a Novel Medium AccessStrategy for LoRa in Industry 4.0 Applications," IECON 2018 - 44th Annual Conference of the IEEE Industrial Electronics Society, Washington, DC, 2018, pp.4141-4146.
[3] S. Sendra, P. Romero-Díaz, J. Navarro-Ortiz și J. Lloret, „Smart Infant Incubator Based onLoRa Networks," 15th International Conference on Computer Systems and Applications (AICCSA), Aqaba, 2018, pp. 1-6.

[4] Semtech Company (LoRA). [Interactiv] 7 February 2020. https://www.semtech.com/lora/why-lora.

[5] A. Zanella, N. Bui, A. Castellani și L. V. a. M. Zorzi, „Internet of Things for Smart Cities," IEEE Internet of Things Journal, vol.I, pp. 2232,2014 .

[6] L. Atzori, A. Iera şi G. Morabito, „Internet of Things security: A survey," Computer Networks: The International Journal of Computer and Telecommunications Networking, vol. LIV, 2010, pp 2787-2805. DOI:https://doi.org/10.1016/j.comnet.2010.05.010.

[7] A. Herutomo, M. Abdurohman, N. A. Suwastika, S. Prabowo și C. W. Wijiutomo, „Forest firedetection system reliability test using wireless sensor network and OpenMTC communication platform," 2015.

[8] J. Toledo-Castro, P. Caballero-Gil, N. Rodríguez-Pérez, I. SantosGonzález şi C. H.-G. a. R.Aguasca-Colomo, „Forest Fire Prevention, Detection, and Fighting Based on Fuzzy Logic and Wireless Sensor Networks".

[9] A. K. Sharma, M. F. R. Ansari, M. F. Siddiqui și M. A. Baig, „IoT Enabled Forest Firedetection and Online Monitoring System," International Journal of Current Trends in Engineering \& Research (IJCTER), Volume 3, Issue 5, pp50-54, 2017.

[10] K. Jayaram, K. Janani, R. Jeyaguru și R. K. a. N. Muralidharan, „Forest Fire Alerting SystemWith GPS Co-ordinates Using IoT," 2019 5th International Conference on Advanced Computing \& Communication Systems (ICACCS), pp 488-491, 2019.

[11] H. Soliman, K. Sudan și A. Mishra, „A Smart Forest-Fire Early Detection Sensory System:Another Approach of Utilizing Wireless Sensor and Neural Networks", in Sensors, 2010 IEEE. IEEE, pp. 19001904, 2010.

[12] M. Hariyawan și A. G. a. E. Putra, ,Wireless Sensor Network for Forest Fire Detection," vol. II, 2013.

[13] G. Hristov, J. Raychev şi D. K. a. P. Zahariev, „Emerging methods for early detection of forestfires using unmanned aerial vehicles and LoRaWAN sensor networks," 2018.

[14] L. Yu, N. Wang și X. Meng, „Real-time Forest Fire Detection with Wireless Sensor," pp1-4, 2005.

[15] R. Vega-Rodríguez, S. Sendra, J. Lloret și P. R.-D. a. J. L. GarciaNavas, „Low Cost LoRa based Network for Forest Fire Detection," 2019.

[16] „Pycom,” [Interactiv]. Available: https://pycom.io/product/lopy4/. [Accesat 15 February 2020].

[17] „Sparkfun,” [Interactiv]. Available: https://www.sparkfun.com /products/14675. [Accesat 9February 2020].

[18] „Arduino,” 25 February 2020. [Interactiv]. Available: https://store. arduino.cc/arduino-mega-2560-rev3.

[19] „Wikipedia,” 29 February 2020. [Interactiv]. Available:https://en. wikipedia.org/wiki/Flame_detector.

[20] A. Lavric și A. 1. Petrariu, „LoRaWAN Communication Protocol: The New Era of IoT," Conference: 2018 International Conference on Development and Application Systems (DAS), 2018, pp 74-77.

[21] A. Lavric, V. Popa, „A LoRaWAN: Long Range Wide Area Networks Study", 11-th International Conference on Electromechanical and Power Systems, pp. 435-438, 2017.

[22] O. Khutsoane și B. I. a. A. M. Abu-Mahfouz, „IoT devices and applications based onLoRa/LoRaWAN," IECON 2017 - 43rd Annual Conference of the IEEE Industrial Electronics Society, Beijing, pp. 6107-6112, 2017.

[23] B. Sarwar, I. S. Bajwa, N. Jamil și S. R. a. N. Sarwar, „An Intelligent Fire Warning ApplicationUsing IoT and an Adaptive Neuro-Fuzzy Inference System," Sensors 19(14):3150, doi:10.3390/s19143150, 2019. 